\title{
The Teacher as Learner: Professional Development Programs as Agents for Change
}

\author{
Penelope Little, Margaret McMillan \\ School of Nursing and Midwifery, University of Newcastle, Australia
}

Purpose: To provide a rationale for the design and implementation of a Professional Development Program for academics and present reflections on the process and outcomes provided by the program facilitators and participants.

Methods: The program methodology centres on a collaborative approach to professional development. However, the focus is on student-centred rather than teacher-centred learning approaches in a manner consistent with the ideals of practice/ problem-based learning philosophy and methodology.

Results: Reflective pieces provided at the conclusion of the program demonstrated that participants satisfied the criteria for leaner-managed learning outcomes.

Conclusion: The context and culture of the academic environment has continuously changed over the last three decades mainly given developments in technology. However, in contexts reliant on technology enhanced learning and teaching, successful on-line learning events still need to reflect a student-centred approach. In most instances professional development opportunities should allow academics the freedom to pursue answers to their own development needs.

Keywords: Professional development; Student-centred learning; On-line learning

\section{INTRODUCTION}

Australian University websites highlight commitment to learning and teaching. Frequently there is also reference to strong support for academics through professional development in pursuit of progressive and good practice, scholarly ideals in learning and teaching and curriculum development. Englund, Olofesson \& Price (2017) suggested that teachers' worldviews around teaching are central to curriculum implementation involving educational technologies in higher education. The senior teachers experienced the greatest difficulty in changing. Less experienced teachers had fewer preconceptions of teaching responsibilities and thus were more likely to embrace innovation and be more student-centred. Hence the transformational potential for educational technology might be limited.

The context and culture of the academic environment has continuously changed over the last three decades mainly given developments in technology (Cook \& Ellaway, 2015). However, in contexts reliant on technology enhanced learning and teaching, successful on-line learning events still need to reflect a student-centred approach; these elements have implications for academics as they strive to develop and maintain optimal learner management systems and processes. Any changes in models of learning has implications for consistent changes in models of working in a learning environment. The latter demands support through professional development that showcases the philosophy and methodology. Delgaty (2013) recommendations included careful appraisal of timing of desirable support interventions, investment in training for academ-

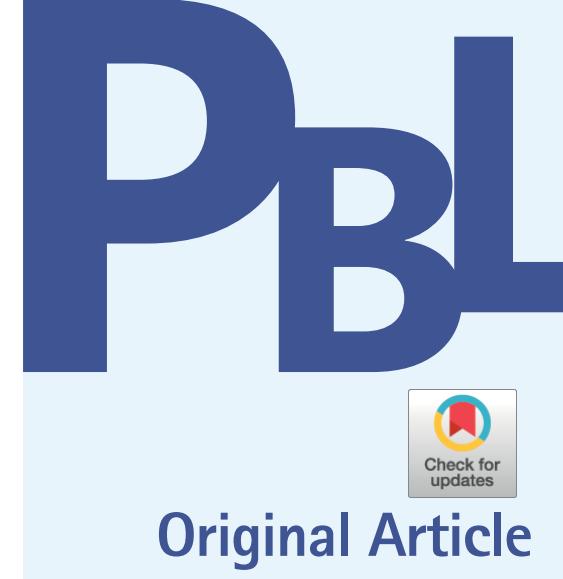

pISSN 2288-8675 · elSSN 2508-9145

J Probl Based Learn 2019;6(2):59-66

https://doi.org/10.24313/jpbl.2019.00185

Received: October 17, 2019

Revised: October 20, 2019

Accepted: October 26, 2019

Corresponding author:

Margaret McMillan

School of Nursing and Midwifery, University of Newcastle, 66 Rickard

Street, Bateau Bay, NSW 2261,

Australia

Tel: +61-408-431269

Fax: +61-243-494538

E-mail: margaret.mcmillan@

newcastle.edu.au
(C) Copyright 2019 International Society for Problem-Based Learning

(c) This is an Open Access article distributed under the terms of the Creative Commons Attribution Non-Commercial License (http://creativecommons.org/licenses/ by-nc/4.0/) which permits unrestricted non-commercial use, distribution, and reproduction in any medium, provided the original work is properly cited. 
ics to focus on their role when reliant on e-learning. consideration of both academic and administrative issues and being vigilant about technical support. This paper describes the design and implementation of one program that has been applied in a variety of higher education contexts, using different delivery methods for groups of different sizes; these included face to face, blended learning and all on-line. Despite the different contexts and delivery methods, the educational approach is maintained through application and modelling of the principles of student-centred learning.

\section{METHODS}

The program methodology centres on a collaborative approach to professional development; the focus is on student-centred rather than teacher-centred learning approaches (McMillan, 2017), but the teacher identifies their own learning needs. The stimuli for learning need to be initiated with student engagement in mind; the material should aim to provoke students to see alternative responses to situations thus engaging in relevant enquiry processes that meets their learning outcomes.

It is important at the outset to allow the group members as learners to identify their own learning needs. Our assumptions in this collaborative learning venture were that the participants have:

- A demanding workload and would be strategic in their approach to meeting the PDP requirements

- Some knowledge and experience of innovative student-centred teaching approaches

- Some knowledge of appropriate teaching/learning evaluation protocols and research methodologies

- Developed some insights into their teaching practice.

- An expectation that the PDP would model a student-centred approach to teaching

The participants learn about and share personal knowledge and experiences of student-centred teaching models in order to increase effectiveness as facilitators of student learning and in encouraging students to take increasing responsibility for their own learning. The approach has been applied by the facilitators to many and varied groups of academics wanting to enhance their own teaching with a stronger focus on student-centred learning. While the principles and main learning activities remain a constant, the delivery method has varied over time and circumstance.

Two examples are reported: The first a course unit undertaken as part of a Tertiary Teaching Award, delivered through three workshops, online forums and individual online facilitation; the second through three synchronous video group sessions, three individual video sessions and online facilitation both group and indi- vidual. The latter is still in progress, however, ongoing reflection by the facilitators provides insights that confirm the methodology is consistent in its potential to bring about change in the teaching approaches of academics.

\section{Rationale}

The Professional Development Program (PDP) allows the participants to explore strategies that may be used to facilitate and enhance student learning based on student-centred methods. The program resources emerge from and capitalize on the wealth of experience from the facilitators themselves; the central concepts deriving from problem/practice/enquiry-based learning and similar student and context-based educational strategies. We hope that this article provides a framework for professional development, particularly as demands for blended on-line learning emerge.

\section{Program objectives}

During the course the participants are encouraged to:

1. Share, discuss and critique student-centred approaches to teaching and learning focusing on particular educational needs and imperatives.

2. Select, develop and implement a student-centred intervention in units coordinated by the participants to enhance student learning outcomes.

3. Evaluate the effectiveness of implementing their proposed teaching/learning intervention/s.

\section{Processes and content}

The participants are asked to identify areas for improvement that are of interest to them but particularly of value for achievement of optimal student learning outcomes. Therefore, the following are key aspirations for pursuit of student-centred learning as a major driver of innovation:

- Review of student-centred methods currently in use within the units

- Identification of opportunities to enhance learning outcomes for students.

- Selection of potential interventions to enhance student learning outcomes.

- Design and conduct a proposal to implement and evaluate the proposed intervention.

- A reflective practice assignment documenting insights gained from program participation

Given their appreciation of the current higher education context in Australia and the participants' workplaces, to inspire thinking around possibilities for small scale quality improvement projects, 
the PDP facilitators suggested areas that might present an opportunity for development:

- Use of relevant stimulus material to provide a context for learning

- Student self-direction around learning

- Student engagement

- Enquiry and clinical decision-making

- The centrality of critical thinking

- Communication strategies

- Assessment including marking and moderation

- Other opportunities to excel as teacher/facilitator/coordinators.

\section{Teaching/delivery methods}

Following consideration of the participants' commitments to their own workplace learning events and curriculum development, negotiations around PDP implementation led to finalization of a plan that would best accommodate opportunities for whole group involvement, peer interaction and individual sessions. Processes and learning activities involved video-conference sessions, online discussion and individual PDP project work. The following guidelines for completion of the PDP were provided:

1. Select either one or more of the course units you are coordinating this semester. Identify teaching strategies designed to align with student-centred learning. Identify one or more areas for enhancement of alignment with student-centred learning.

2. Areas for enhancement: Identification of your choice of project should articulate the student learning need or opportunity for improvement being addressed. Changes to teaching strategies are usually made in response to some perceived lack in student learning outcomes and a new or enhanced teaching strategy is adopted. You will need to describe and defend the selected teaching/learning strategy and consider the implications for the students and your peers as well as curriculum implementation.

3. Program/Course context: In addition to identifying the program and course of study, information about the numbers of students, place of course in program, broad aims of course and other relevant information should be included.

4. Alignment with principles of student-centred teaching: The teaching/learning strategy should be analysed in terms of its congruence with the principles of student-centred learning approaches. Each element of the strategy should be described in terms of its capacity to evidence one or more of the principles.

5. Anticipated outcomes: These may be described in terms of specific learning outcomes for students that can be evidenced in assessment and/or student evaluations. The outcomes should be attributable wholly or in part to the intervention.

6. Specific teaching/ learning strategy or intervention: This should describe the specific elements of the strategy and its implemen- tation (i.e. the learning activity or assessment task should be described in detail). If it is a group task, you need to show what guidelines are given, numbers of group members, whether product and process are documented.

7. Proposed evaluation protocol: This identifies the specific methods of data collection you have selected to evaluate the effectiveness of the strategy and/or its achievement of the anticipated outcomes.

Following acceptance of the above guidelines, the program facilitators then provided their own principles, objectives, assumptions and rules of engagement in an effort to ensure the maximum level of collaboration among all members of the group.

The following is an overview of the principles, objectives, assumptions and rules of engagement.

\section{Principles}

The Professional Development Plan and its implementation allow participants to:

- Recognise the participants as professional colleagues with relevant experience and expertise

- Operate from a framework and philosophy that focuses on student-centred learning approaches and context-based learning

- Model a student-centred approach and context-based learning

- Provide appropriate activities to enable the participants to demonstrate their capabilities

- Provide appropriate feedback in a timely manner

- Model reflective practice using relevant, structured frameworks. Our objectives were as follows:

To ensure the participants were:

- Able to use their current and or proposed teaching as a focus for the learning/teaching enhancement project and thus integrate the project into their workload.

- Provided with the opportunity to interact as professional colleagues with each other and with the facilitators.

- Challenged to expand their understanding of the relationship between a specific teaching activity and student learning outcomes.

- Extended in terms of understanding of student-centred learning and teaching.

- Provided the opportunity to experience the role of a student in a student-centred approach to teaching through the PDP

- Encouraged to use some relevant frameworks for critical reflection on their practice

- Facilitated to produce a proposal for enhancement of the course unit/s they are teaching/ coordinating that could be implemented this semester or in a future offering. 
Some rules of engagement for the individual sessions:

- Confidentiality is respected by both parties.

- Either party may call Time Out to discuss the process of the engagement as opposed to the content.

- Participants will take responsibility for ensuring the PDP is meeting their needs.

- Facilitators will seek feedback from the participant on the relevance of the program to the participant.

- The PDP will structure activities related to the participant's current coordination/teaching role to provide opportunities for application of a student-centred approach to teaching and learning.

- The participant will engage with agreed activities and provide feedback to facilitators.

- The schedule of individual meetings will have agenda items proposed by both facilitators and participant.

- Feedback on group sessions will be provided to participants and feedback on individual sessions will be provided from both facilitators and participant on the progress of participant's PDP.

Some rules of engagement for group sessions

- Facilitators will provide an agenda/outline of the session in advance.

- Participants may be required to prepare and present to the group some element of the participant's PD Plan or activities.

- Participants will be expected to engage with each other and the facilitators in the group sessions as peer learners and colleagues.

- Group sessions will be an opportunity for discussion, clarification and exploration.

The facilitators provided the following guidance on expectations for processes of reflection (by both facilitators and group members) that is an important part of any learning opportunity and should be both formative and summative:

Participants will be encouraged to reflect formatively on the content and process of the PD program throughout the course to better guide the implementation of the Program and ensure its relevance to all participants.

In addition, participants will be invited to complete a summative reflective piece (a Reflection on Practice) at the conclusion of the Program.

This PDP item requires personal reflection on your experience in the Program and to documentation of your insights.

Reflections could include insights on:

- Specific enhancements of teaching and learning strategies employed and their impact on student learning

- The impact of the context (challenges/enhancers) in implementing enhancements and using student-centred teaching methods

- The opportunities for enhancing scholarship of teaching in relation to your teaching role.
- Your experience as a participant in the PDP in relation to the implementation of student-centred teaching methods-Documentation of reflections should meet the following criteria:

1. Reflections include insights from a range of experiences in the PDP.

2. Experiences are analysed in terms of relevant theoretical frameworks.

3. Insights are discussed in terms of alignment with personal philosophy of teaching and learning and/or a conceptual framework of teaching and learning

4. Outcomes are discussed in terms of impact on further development of approaches to teaching and learning

\section{RESULTS}

All participants and the program facilitators completed a summative reflection guided by criteria presented at the commencement of the program. Two pieces of reflection were provided after formal consent for publication was obtained from four participants who could be contacted for consent. The following are the reflections from one participant and the course facilitators. In addition, three emergent themes were evident in formal interactions from group and individual sessions.

\section{Participant from award program}

\section{As a student}

Having been away from a classroom as a learner for a long time, it was a struggle getting back into the student role that was expected all of us to be - an actively involved student in a teaching course! I have envisioned myself to be lost in the midst of non-science oriented colleagues. Nevertheless, I survived the first workshop and realized that I actually had something to contribute in the discussion and that my teaching experience was my greatest source of knowledge.

The first hurdle I had to get over with as a student in this course was my pre-conceived notion that science teaching cannot be and does not have to be student-centred and it would not be possible to align it with the principles of student-centred learning. Thus, planning a teaching/learning intervention was a struggle. Much more, I had the feeling that most of the course participants were also, like me, lost. During the reversal of role exercise in the first workshop, I felt that most of us were comfortable being in the learner situation but were having difficulty as facilitator and resource person - more so, myself. I got out of the first workshop not really sure of what I was going to do and not fully believing that the student-centred approach is the way to go in science teaching.

The project proposal preparation was an enriching learning ex- 
perience for me because it was during this stage that I actually became convinced that I could drive my teaching strategy towards more student-centred approaches. Comments on the proposal, especially the first two drafts, were very helpful and clarified a lot of issues associated with the project implementation and evaluation. The support of my critical friend was likewise invaluable.

I am of the view that even if the group didn't have regular face to face contact with our facilitator, we were continually learning all throughout the semester. The project taught me a lot about student-centredness in teaching. It was after all a student-centred approach of learning by itself. I was involved, it was relevant to my work, it transformed my teaching.

Last Monday's culminating workshop was very rewarding. It was like the group was bonded as one, talking about each other's project, offering suggestions. I sensed that everybody was comfortably relating to student-centred teaching, gone were the apprehensions, uncertainty and the questioning looks. I myself felt that way.

My learning experience in the course is invaluable. Were the course objectives fulfilled? Definitely! I learned about student-centred teaching and much more, I applied the approach to my discipline area, I evaluated its effectiveness and finally I became convinced that this is the approach I would like to utilise for all my teaching to promote active learning. [P1 science teacher]

\section{As a Teacher Innovator}

The chosen project was implemented on a course that I taught for the first time utilizing a new textbook and operating under a new set-up, i.e. nine weeks of lecture instead of 12 for the same amount of materials. It was just the right time to introduce a new teaching strategy.

I sensed that, initially, the students were not so receptive to the idea of activity worksheets. While the first three 'lectorials' went well, it was a challenge to get the students to participate in the first hour. I persevered, I gave them more time, I enthused them, and finally it paid off. They warmed up to the idea and started to be more involved, they asked more questions and clarifications and longer discussions took place. Before thethree 3 hours was over, they were already talking about the effect of the formative assessment to their learning. The next two weeks went very well and the lectures were never dull. It was so gratifying to see the lit faces of the students after the 'lectorials.

All throughout the intervention, I was dealing with the conflict between course demands and time, thus, the discussion was sometimes rushed. In addition, the set-up of the 'lectorials' (all in one day, two hours followed by another hour with a one hour break in between) didn't allow adequate time for the students to process the given information. However, most of them quickly adapted to the pace of the 'lectorial'.

I am most pleased that the students, according to their SET (student evaluation of teaching) responses, actually felt that I, through my teaching intervention, (1) motivate them to extend their learning and (2) help them understand the importance of the content to their program. These are the two aspects of my teaching that I have been working to improve on and seem to be more transparent in student-focused learning strategies such as the 'lectorial'.

Central to my teaching philosophy is my strong belief that the primary regulator of students' learning and motivation is teacher-student interaction. It is my view that my positive interaction with the students contributed significantly to the success of the intervention. They were more willing to give me a chance and more willing to participate because I have established good rapport with them. I believe that in order to ensure favourable responses to any type of student-centred learning strategy, it is important for the teacher to first establish good rapport with the class. [P4, Award program]

\section{The Project: From Beginning to End}

I was challenged by this project! But I learned a lot from it.

I did take in what the facilitator has said during the first workshop that it is important to get the proposal right. And so I worked hard to get it right and it became my point of reference. I found that the proposal gave me focus especially during the preparation of the poster report and the analysis of the evaluation results. It was also very helpful that we were given the report criteria.

While the project took a lot of my time otherwise devoted to research, I don't regret spending the time on enhancing my teaching. I gained a better understanding of student-centred learning approaches and how I can apply them to my discipline; its implementation gave me the chance to enhance my teaching and interaction with students; the report helped me to consolidate my results. Overall, the project convinced me that student-centred approaches can work in science teaching.

Finally...The project successfully transformed me and my teaching. I don't believe that I can go back to being a 'normal' science lecturer. My 'lectorial' teaching experience has given me the motivation to make my teaching more relevant to the students' needs. I will, however, be realistic - start small and simple. Perhaps, my next project will be the use of 'lectorial' approach and formative tests in big 'foundation' classes. First year students certainly will benefit from the set-up given adequate time and proper class management. [P 4, Award program]

In a similar fashion to the participants, the consultant/s provided their reflections on the issues identified by participants and summaries of the project proposals. 


\section{Reflection: The Facilitator of the award program}

Before providing some reflective comment I should reiterate my objectives and some assumptions outlined above in the description of the Professional Development Program

My reflections on achievement of objectives

The course met my objectives in that I believe the opportunities identified were provided to participants. The extent to which participants used the opportunities varied considerably as I would expect in any student population. My observation is that those who used the opportunities got the most out of the course. This is consistent with my experience of student-centred approaches to teaching.

All projects reflected some degree of enhanced implementation of student-centred teaching strategies. The sophistication of the strategies in the projects varied and reflected the experience of the participants in tertiary education.

\section{Course design and delivery}

I had designed the course so that it models my understanding of student-centred teaching and thus the content of the course is the design of the learning experiences. Some participants have challenged my assumptions about participants having some knowledge and experience of student-centred teaching arguing that as new to the tertiary sector, they had little understanding of this concept. However, all participants did have experience of student-centred teaching (e.g. as doctoral students themselves or in other contexts) and were able to contribute effectively to discussion of the principles in Workshop 1. However, some stated that they struggled to find an agreed definition and wanted some further input from the facilitator, especially in Workshop 1. While of course it is appropriate to have expert input as a learning opportunity in student-centred teaching, it should, in my view, follow some exploration by the learner. Providing a definition at this stage would pre-empt the engagement of the learner with an examination of their own assumptions and the literature. My observations were that this made some participants uncomfortable and frustrated which also reflects the experiences of their own students when confronted with student-centred teaching strategies. I have learned that while I am the object of that frustration, it is my role as facilitator to provide the direction and guidance but not pre-empt the learning.

In using a student-centred teaching approach I am mindful of the need to support students in their exploration of the learning opportunities and achievement of learning outcomes. I usually structure this support through the provision of explicit guidance and feedback on the learning activities and or assessment tasks. While guidance and feedback was provided on the project proposal, project report and final poster presentation, the group and the products would have benefited from possibly breaking down the project report into parts for earlier draft presentations. e.g. some submission of the relevant literature and conceptual frameworks. I would also require staged submissions of the reflective exercise, using specific areas and frameworks for reflection.

Regarding the assessment, I found the projects to be reflective of student-centred approaches. However, the challenge of designing implementing and evaluating a teaching enhancement project within one semester is considerable, even when attempts are made to ensure the scope of the projects is manageable. As some data collection requires ethics clearance, the projects in the main were pilots for what could be an extended study. This still makes the project requirement useful in my opinion. The posters were very effective and I hope they will be exhibited.

A great challenge for me was the use of 'BlackBoard' [online learning platform] in a student-centred teaching approach. My experience and skills are very limited and I was grateful for the input of some participants who took the initiative in filling in the gaps. I not only need more skill development in the effective use of the system, but need to think much more about how to translate to online, strategies I have used with effect in workshops to engage participants. My observations are that few participants used the online opportunities to interact with each other or with the facilitator. This is despite the fact that many participants acknowledged in their reflections that they were experiencing confusion and lack of confirmation that they were on the right track. This also seems to be a common experience of participants with their own students in online teaching. I would really like some assistance from participants as to what might have improved this area of the course.

My personal learning journey has been enriched by the challenge of working with participants from different disciplines and in different teaching/learning contexts. I am always appreciative of the opportunity to share teaching experiences with my colleagues and realize how much I enjoy the role of facilitator. Thank you for your participation and I look forward to your comments on the course.

\section{Emergent themes in records of individual/group interac- tions: non-award program}

Three major themes emerged as central issues of concern from reports from participants (facilitators and academics) on their experience of the learning processes in the non-award program ie not part of a formal Graduate Award: The value of modelling student centred-learning; the context of practice as a learning resource; and peer learning.

\section{The value of modelling student centred-learning}

At all times the consultants were conscious of modelling the centrality of the concept of putting the learner at the centre of 
learning events; they provided their reflections on the issues identified by participants and summaries of the project proposals. $\mathrm{Ob}$ servations provided by participants indicated that the consultants' approach did not involve a high level of input of content e.g. educational theories. This was intentional with goal being to create a level of discomfort that mirrors student experiences and incites a level of 'cognitive dissonance' that ultimately promotes engagement and responsibility for self-directed learning. The stimulus for learning was the requirement for individual project proposals to involve identification of an area for improvement and then development and implementation of an intervention to address the issue.

Both the project proposal and implementation as well as the reflective practice exercise could be used in an award course as assessment activities and these essentially acted as the driver for all development activities.

The consultants elaborated on the goals for reflection on learning and/or practice and the extent which students are caused to fully explore their thinking, beliefs, feelings. It was suggested that ways to encourage meaningful reflection is to include exercises such as testing assumptions at the outset of the course unit and revisiting these assumptions after peer to peer (or group) interactions.

The consultants emphasised that it is also necessary to provide different frameworks for students to use in the reflective process. These might include theoretical frameworks; practice frameworks such as therapeutics; scope of practice, regulatory policies; legal and ethical frameworks; quality and safety frameworks etc.

By constantly questioning choices in designing learning events one can ask 'What am I actually asking the students to do?' i.e. Questioning the nature and rationale for choices around intentions for student learning should confirm the learning event as a means of active engagement and requiring application to practice.

It was noted that some programs use assessment as the major driver for all learning activities. This aligns with the evidence that students are motivated by the assessment and act strategically to meet those requirements. However, assessment should align with the content of the unit and focus on testing application to practice as well as provide clear and relevant evidence for achievement of specified learning outcomes and graduate competencies/capabilities. Percentages attributed to group tasks and ways to differentiate among individual contributions were discussed. Tasks that involve individual reflection were seen as worthy. It was noted that appreciation by students of the rationale for group work was critical.

\section{The context of practice as a learning resource}

Staff have a great deal to bring to the program and units through their extensive clinical experience as clinicians, clinical educators and educators. This experience is a valuable capability that could be used to ensure the relevance of the content and learning activities to the students and to provide the kind of practice-based stimulus for learning that is a key element in an instructional strategy that ensures students have a relevant context in which to interrogate and apply new learning ie the norm for traditional approaches is theory applied to practice; context-based learning suggests a Practice-Theory-Practice sequence.

In a similar way to the tenets of Work-integrated Learning, the academic workplace provides a 'stimulus for learning' that creates 'a need to know' about processes and systems that are new to the academic.

Participants expressed a sense of discomfort around an apparent set of explicit directions on project choices. The consultants suggested that 'situations' that encourage learning need to 'confront' and 'disconcert' - In this way they, like their own students are provoked to think.

There was a need to challenge long held beliefs about the value of for example face-to face sessions being more likely to lead to more substantial learning. It was noted by facilitators that like them, the students' reliance on their own life experiences and belief systems inform their responses, decisions and actions: There was a need for the facilitator/teacher to interrogate these for validity as the students pursue self-directed learning.

\section{Peer learning}

In a similar way to typical student-centred classroom practices, participants were encouraged in group sessions to respond to issues raised by their peers; they engaged in a high level of peer-peer interaction in both sessions and provided feedback that they valued this opportunity highly.

Encouragement of student-oriented interactions among peers, by exploring a way to embrace peer to peer learning, especially those reliant on self-direction in learning, is a worthy pursuit. This necessitated some thinking around the purpose of student on-line forums and ways to integrate other elements of the unit into the forum activities.

The academic environment was the context for learning; this provided the potential to work with peers just as one might with their own student group/s. A focus on their own work roles enabled participants to choose a 'critical friend' to test ideas around any review of current course units being delivered (taught/coordinated). For example, consideration of the value of choosing the timing of a practice scenario required investigation. In response to a query from an academic, the consultants discussed the benefits of using the scenario earlier in the unit before engagement with theory as a means of having students identify what they already know and what they need to learn in order to respond to the situa- 
tion. It was suggested that participants might consider reviewing the use and placement of cases, pointing out the value of the case as the initial learning stimulus in the unit/topic as a framework for of the interrogation of the presented concepts and content. The initial response of the students to the case should then be revisited in the light of the further learning (self, peer, facilitated and expert presentation).

The consultants talked about the provision of stimulus material suited to differing professional roles and the need to cause students to engage with the situation and interact with others. They noted that it was all about 'relevance for students' and the importance of recognizing the extent to which design impacts on learning processes that are meaningful to different professional roles.

In individual sessions exploration of ideas and strategies centred on the value that derives from well designed 'group work' where students interact with each other, sessions are not recorded but the group nominee is responsible for feedback to the facilitator; this seemed particularly useful for 'asynchronous' sessions in blended learning situations involving choices in online and face to face tutorials. The advantages and limitations of peer learning activities, their purpose, mechanisms for assessment and ways to overcome perceived disadvantages were discussed.

\section{CONCLUSION}

This paper provides an insight as well as a template for working with experienced teachers to enhance their confidence with stu- dent-centred learning. The context and culture of the academic environment has continuously changed over the last three decades mainly given developments in technology. However, in contexts reliant on technology enhanced learning and teaching, successful on-line learning events still need to reflect a student-centred approach. In most instances professional development opportunities should model a student-centred approach and allow academics the freedom to pursue answers to their own development needs.

\section{ACKNOWLEDGEMENTS}

None.

\section{REFERENCES}

Cook, D.A., \& Ellaway, R.H. (2015). Evaluating Technology Enhanced Learning: A Comprehensive Framework. Medical Teacher, 37(10), 961-970.

Delgaty, L. (2013). A Critical examination of the time and workload involved in the design and delivery of an e-module in postgraduate clinical education. Medical Teacher, 35(5), e1173-1180.

Englund, C., Olofsson, A. D., \& Price, L. (2017). Teaching with technology in higher education: understanding conceptual change and development in practice. Higher Education Research \& Development, 36(1), 73-87.

McMillan, M. (2017). Towards Student-Centred Learning Activities. Journal of Problem-based Learning, 4(1), 1-4. 